\title{
Approximating Minimum Manhattan Networks in Higher Dimensions
}

\author{
Aparna Das ${ }^{1}$, Emden R. Gansner ${ }^{2}$, Michael Kaufmann ${ }^{3}$, Stephen Kobourov ${ }^{1}$, \\ Joachim Spoerhase ${ }^{4}$, and Alexander Wolff ${ }^{4}$ \\ 1 Dept. of Comp. Sci., University of Arizona, Tucson, AZ, U.S.A. \\ 2 AT\&T Labs Research, Florham Park, NJ, U.S.A. \\ 3 Wilhelm-Schickard-Institut für Informatik, Universität Tübingen, Germany \\ 4 Institut für Informatik, Universität Würzburg, Germany
}

\begin{abstract}
We study the minimum Manhattan network problem, which is defined as follows. Given a set of points called terminals in $\mathbb{R}^{d}$, find a minimum-length network such that each pair of terminals is connected by a set of axis-parallel line segments whose total length is equal to the pair's Manhattan (that is, $L_{1^{-}}$) distance. The problem is NP-hard in 2D and there is no PTAS for 3D (unless $\mathcal{P}=\mathcal{N} \mathcal{P}$ ). Approximation algorithms are known for $2 \mathrm{D}$, but not for $3 \mathrm{D}$.

We present, for any fixed dimension $d$ and any $\varepsilon>0$, an $O\left(n^{\varepsilon}\right)$-approximation algorithm. For 3D, we also give a $4(k-1)$-approximation algorithm for the case that the terminals are contained in the union of $k \geq 2$ parallel planes.
\end{abstract}

Keywords: Approximation Algorithms, Computational Geometry, Minimum Manhattan Network

\section{Introduction}

In a typical network construction problem, one is given a set of objects to be interconnected such that some constraints regarding the connections are fulfilled. Additionally, the network must be of little cost. For example, if the objects are points in Euclidean space and the constraints say that, for some fixed $t>1$, each pair of points must be connected by a path whose length is bounded by $t$ times the Euclidean distance of the points, then the solution is a so-called Euclidean $t$-spanner. Concerning cost, one usually requires that the total length of the network is proportional to the length of a Euclidean minimum spanning tree of the points. Such low-cost spanners can be constructed efficiently [2].

In this paper, we are interested in constructing 1-spanners, with respect to the Manhattan (or $L_{1^{-}}$) metric. Rather than requiring that the total length of the network is proportional to the minimum spanning tree of the points, our aim is to minimize the total length (or weight) of the network. Note that the Euclidean 1-spanner of a set of points is simply the complete graph (if no three points are collinear) and hence, its weight is completely determined. Manhattan 1-spanners, in contrast, have many degrees of freedom and vastly different weights.

More formally, given two points $p$ and $q$ in $d$-dimensional space $\mathbb{R}^{d}$, a Manhattan path connecting $p$ and $q$ (a $p-q M$-path, for short) is a sequence of axis-parallel line segments connecting $p$ and $q$ whose total length equals the Manhattan distance between $p$ and $q$. Thus an M-path is a monotone rectilinear path. For our purposes, a set of axis-parallel line segments is a network. Given a network $N$, its weight $\|N\|$ is the sum over the lengths of its line segments. A network $N$ Manhattan-connects (or M-connects) two given points $p$ and $q$ if it "contains" a $p-q$ M-path $\pi$. Note that we slightly abuse the notation here: we mean pointwise containment, that is, we require $\bigcup \pi \subseteq \bigcup N$. Given a set $T$ of points - called terminals - in $\mathbb{R}^{d}$, a network $N$ is a Manhattan network (or $M$-network) for $T$ if $N$ M-connects every pair of terminals in $T$. The minimum Manhattan 


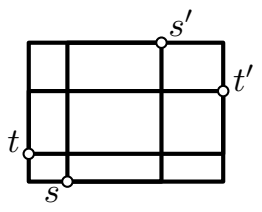

(a) an M-network for $T=\left\{s, s^{\prime}, t, t^{\prime}\right\}$

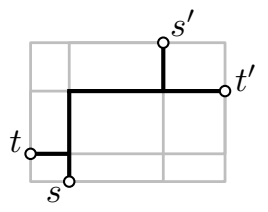

(b) a minimum Mnetwork for $T$

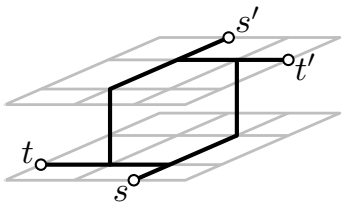

(c) a minimum $\mathrm{M}$ network in $3 \mathrm{D}$

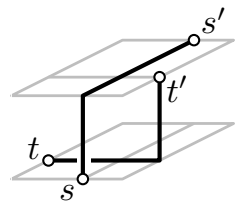

(d) M-paths missing each other

Fig. 1: Examples of M-networks in 2D and 3D.

network problem (MMN) consists of finding, for a given set $T$ of terminals, a minimum-weight M-network. For examples, see Fig. 1.

M-networks have important applications in several areas such as VLSI layout and computational biology. For example, Lam et al. [12] used them in gene alignment in order to reduce the size of the search space of the Viterbi algorithm for pair hidden Markov models.

\subsection{Previous work}

The 2D-version of the problem, 2D-MMN, was introduced by Gudmundsson et al. [9]. They gave an 8- and a 4-approximation algorithm. Later, the approximation ratio was improved to 3 [38] and then to 2, which is currently the best possible. It was achieved in three different ways: via linear programming [5], using the primal-dual scheme [15] and with purely geometric arguments [10]. The last two algorithms run in $O(n \log n)$ time, given a set of $n$ points in the plane. A ratio of 1.5 was claimed [16], but apparently the proof is incomplete [8]. Chin et al. 6] finally settled the complexity of 2D-MMN by proving it NP-hard.

A little earlier, Muñoz et al. [14] considered 3D-MMN. They showed that the problem is NP-hard and that it is NP-hard to approximate beyond a factor of 1.00002. For the special case of 3D-MMN, where any cuboid spanned by two terminals contains other terminals or is a rectangle, they gave a $2 \alpha$-approximation algorithm, where $\alpha$ denotes the best approximation ratio for $2 \mathrm{D}-\mathrm{MMN}$. They posed the design of approximation algorithms for general 3D-MMN as an open problem.

\subsection{Related problems}

As we observe in Section 2.3, MMN is a special case of the directed Steiner forest problem (DSF). More precisely, an instance of MMN can be decomposed into a constant number of DSF instances. The input of DSF is an edge-weighted directed graph $G$ and a set of vertex pairs. The goal is to find a minimum-cost subgraph of $G$ (not necessarily a forest) that connects all given vertex pairs. Recently, Feldman et al. [7] reported, for any $\varepsilon>0$, an $O\left(n^{4 / 5+\varepsilon}\right)$-approximation algorithm for DSF, where $n$ is the number of vertices of the given graph. This bound carries over to $d \mathrm{D}-\mathrm{MMN}$.

An important special case of DSF is the directed Steiner tree problem (DST). Here, the input instance specifies an edge-weighted digraph $G$, a root vertex $r$, and a subset $S$ of the vertices of $G$ to which $r$ must connect. An optimum solution for DST is a minimum-weight $r$-rooted subtree of $G$ spanning $S$. DST admits an $O\left(n^{\varepsilon}\right)$-approximation for any $\varepsilon>0$ [4].

A geometric optimization problem that resembles MMN is the rectilinear Steiner arborescence problem (RSA). Given a set of points in $\mathbb{R}^{d}$ with non-negative coordinates, a rectilinear Steiner arborescence is a spanning tree that connects all points with M-paths to the origin. As in MMN, the 
aim is to find a minimum-weight network. For 2D-RSA, there is a polynomial-time approximation scheme (PTAS) [13] based on Arora's technique for approximating geometric optimization problems such as TSP [1]. It is not known whether 2D-MMN admits a PTAS. Arora's technique does not directly apply here as M-paths between terminals forbid detours and thus may not respect portals.

\subsection{Our contribution}

We first present a $4(k-1)$-approximation algorithm for the special case of 3D-MMN where the given terminals are contained in $k \geq 2$ planes parallel to the $x-y$ plane; see Section 3 .

Our main result is an $O\left(n^{\varepsilon}\right)$-approximation algorithm for $d \mathrm{D}$-MMN, for any $\varepsilon>0$. We first present the algorithm in detail for three dimensions; see Section 4 . Since the algorithm for arbitrary dimensions is a straightforward generalization of the algorithm for $3 \mathrm{D}$ but less intuitive, we describe it in the appendix.

Our $O\left(n^{\varepsilon}\right)$-approximation algorithm for $d \mathrm{D}$-MMN constitutes a significant improvement upon the best known ratio of $O\left(n^{4 / 5+\varepsilon}\right)$ for (general) directed Steiner forest [7]. We obtain this result by exploiting the geometric structure of the problem. To underline the relevance of our result, we remark that the bound of $O\left(n^{\varepsilon}\right)$ is the best known result also for other directed Steiner-type problems such as DST [4] or even acyclic DST [18].

Our $O(k)$-approximation algorithm for the $k$-planes case relies on recent work by Soto and Telha [17]. They show that, given a set of red and blue points in the plane, one can determine efficiently a minimum-cardinality set of points that together pierce all rectangles having a red point in the lower left corner and a blue point in the upper right corner. Combining this result with an approximation algorithm for 2D-MMN, yields an approximation algorithm for the 2-planes case. We show how to generalize this idea to $k$ planes.

\section{Some Basic Observations}

We begin with some notation. Given a point $p \in \mathbb{R}^{3}$, we denote the $x$-, $y$ - and $z$-coordinate of $p$ by $x(p), y(p)$, and $z(p)$, respectively. Given two points $a$ and $c$ in $\mathbb{R}^{2}$, let $R(a, c)=\left\{b \in \mathbb{R}^{2} \mid\right.$ $x(a) \leq x(b) \leq x(c), y(a) \leq y(b) \leq y(c)\}$ be the rectangle spanned by a and $c$. If a line segment is parallel to the $x-, y$-, or $z$-axis, we say that it is $x^{-}, y^{-}$, or $z$-aligned. In what follows, we consider the 3-dimensional case of the MMN problem, unless otherwise stated.

\subsection{Quadratic Lower Bound for Generating Sets in 3D}

Intuitively, what makes 3D-MMN more difficult than 2D-MMN is the following: in 2D, if the bounding box of terminals $s$ and $s^{\prime}$ and the bounding box of $t$ and $t^{\prime}$ cross (as in Fig. 1b), then any $s-s^{\prime}$ M-path will intersect any $t-t^{\prime}$ M-path, which yields $s-t^{\prime}$ and $t-s^{\prime}$ M-paths for free (if $s$ and $t$ are the lower left corners of their respective boxes). A similar statement for 3D does not hold; M-paths can "miss" each other - even if their bounding cuboids cross; see Fig. 1d.

Let us formalize this observation. Given a set $T$ of terminals, a set $Z$ of pairs of terminals is a generating set [11] if any network that M-connects the pairs in $Z$ in fact M-connects all pairs of terminals. In 2D, any MMN instance has a generating set of linear size [11. Unfortunately this result does not extend to 3D. Below, we construct an instance that requires a generating set of size $\Omega\left(n^{2}\right)$. The idea of using linear-size generating sets is exploited by several algorithms for 2D-MMN [5,11]. The following theorem shows that these approaches do not easily carry over to 3D. 
Theorem 1. There exists an instance of $3 D-M M N$ with $n$ terminals that requires a generating set of size $\Omega\left(n^{2}\right)$.

Proof. We construct an instance that requires a generating set of size at least $n^{2} / 4$. The main idea of the construction is to ensure that $n^{2} / 4$ of the terminal pairs must use an edge segment unique to that specific pair. The input consists of two sets $T$ and $T^{\prime}$, each with $n / 2$ terminals, with the following coordinates: for $0 \leq i<n / 2$, terminal $t_{i} \in T$ is at $(i, n / 2-i, n / 2-i)$ and terminal $t_{i}^{\prime} \in T^{\prime}$ is at $(n / 2+i, n-i, n-i)$. Figure 2 shows the instance for $n=6$.

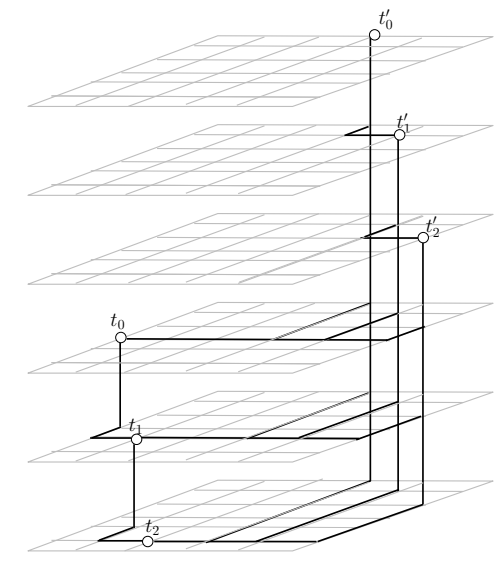

Fig. 2: The constructed network for $n=6$.

Consider any given generating set $Z \subset T \times T^{\prime}$ such that there is a pair $\left(\tilde{t}, \tilde{t^{\prime}}\right), \tilde{t} \in T$ and $\tilde{t}^{\prime} \in T^{\prime}$ that is not in $Z$. We now construct a specific network that contains M-paths between all terminal pairs in $Z$ but no M-path between $\left(\tilde{t}, \tilde{t^{\prime}}\right)$.

Consider any pair $\left(t, t^{\prime}\right) \in Z$ such that $t=(i, j, k) \in T$ and $t^{\prime}=\left(i^{\prime}, j^{\prime}, k^{\prime}\right) \in T^{\prime}$. The M-path from $t$ to $t^{\prime}$ has three segments: an $x$-aligned segment from $(i, j, k)$ to $\left(i^{\prime}, j, k\right)$, a $y$-aligned segment from $\left(i^{\prime}, j, k\right)$ to $\left(i^{\prime}, j^{\prime}, k\right)$, and a $z$-aligned segment from $\left(i^{\prime}, j^{\prime}, k\right)$ to $\left(i^{\prime}, j^{\prime}, k^{\prime}\right)$. To ensure an M-path between each generating pair $t_{i}, t_{j} \in T$ ( similarly between $t_{i}^{\prime}, t_{j}^{\prime} \in T^{\prime}$ ), we add M-paths between each pair of consecutive terminals in $T$ (similarly for $T^{\prime}$ ) as follows: we connect $t_{i}, t_{i+1} \in T$ by adding a $z$-aligned segment from $t_{i}=(i, j, k)$ to $(i, j, k-1)$, a $y$-aligned segment to $(i, j-1, k-1)$, and an $x$-aligned segment to $t_{i+1}=(i+1, j-1, k-1)$; see Fig. 2 .

It is easy to verify that, in this construction, the M-path between terminals $t=(i, j, k) \in T$ and $t^{\prime}=\left(i^{\prime}, j^{\prime}, k^{\prime}\right) \in T^{\prime}$ must use the $y$-aligned segment between $\left(i^{\prime}, j, k\right)$ and $\left(i^{\prime}, j^{\prime}, k\right)$. Since this segment is added only between terminal pairs that are present in the generating set $Z$, there is no M-path between terminals $\tilde{t} \in T$ and $\tilde{t}^{\prime} \in T^{\prime}$ which are not in $Z$. Thus, in order to obtain M-paths between all pairs of terminals in $T \cup T^{\prime}$, we need at least all of the $n^{2} / 4$ pairs in $T \times T^{\prime}$.

\subsection{Hanan Grid and Directional Subproblems}

First, we note that any instance of MMN has a solution that is contained in the Hanan grid, the grid induced by the terminals; see Fig. 1(a). Gudmundsson et al. [9] showed this for 2D; their proof generalizes to higher dimensions. In what follows, we restrict ourselves to finding feasible solutions that are contained in the Hanan grid. 
Second, to simplify our proofs, we consider the directional subproblem of 3D-MMN which consists of connecting all terminal pairs $\left(t, t^{\prime}\right)$ such that $t$ dominates $t^{\prime}$, that is, $x(t) \leq x\left(t^{\prime}\right)$, $y(t) \leq y\left(t^{\prime}\right), z(t) \leq z\left(t^{\prime}\right)$, and $t \neq t^{\prime}$. We call such terminal pairs relevant.

The idea behind our reduction to the directional subproblem is that any instance of 3D-MMN can be decomposed into four subproblems of this type. One may think of the above-defined directional subproblem as connecting the terminals which are oriented in a north-east (NE) configuration in the $x-y$ plane (with increasing $z$-coordinates). Analogous subproblems exist for the directions NW, SE, and SW. Note that any terminal pair belongs to one of these four categories (if seen from the terminal with smaller $z$-coordinate).

The decomposition extends to higher dimensions $d$, by fixing the relationship between $\left(t, t^{\prime}\right)$ for one dimension (for example, $z$ ), and enumerating over all possible relationships for the remaining $d-1$ dimensions. This decomposes $d \mathrm{D}-\mathrm{MMN}$ into $2^{d-1}$ subproblems, which is a constant number of subproblems as we consider $d$ to be a fixed constant.

This means that any $\rho$-approximation algorithm for the directional subproblem leads to an $O(\rho)$-approximation algorithm for the general case. Thus we can focus on designing algorithms for the directional subproblem.

Observation 1 Any instance of $M M N$ can be decomposed into a constant number of directional subproblems. Thus a $\rho$-approximation algorithm for the directional subproblem leads to an $O(\rho)$ approximation algorithm for $M M N$.

\subsection{Relation to Steiner Problems}

We next show that there is an approximation-preserving reduction from directional 3D-MMN to the directed Steiner forest (DSF) problem, which by Observation 1, carries over up to a constant factor, to general $3 \mathrm{D}-\mathrm{MMN}$.

Let $T$ be a set of $n$ points in $\mathbb{R}^{3}$. Let $H$ be the Hanan grid induced by $T$. We consider $H$ as an undirected graph where the length of each edge equals the Euclidean distance between its endpoints. We orient each edge in $H$ so that, for any edge $\left(p, p^{\prime}\right)$ in the resulting digraph $H^{\prime}$, the start node $p$ dominates the end node $p^{\prime}$. We call $H^{\prime}$ the oriented Hanan grid of $T$. Now let $\left(t, t^{\prime}\right)$ be a relevant pair of points in $T$, that is, $t$ dominates $t^{\prime}$. Any M-path in $H$ connecting $t$ to $t^{\prime}$ corresponds to a directed path in $H^{\prime}$ from $t$ to $t^{\prime}$. The converse also holds: every directed path in $H^{\prime}$ corresponds to an M-path in $H$.

Let $I$ be an instance of directional 3D-MMN and let $I^{\prime}$ be an instance of DSF where the input graph is $H^{\prime}$ and where every relevant terminal pair of $I$ has to be connected. Then, each feasible solution $N$ of $I$ contained in $H$ corresponds to a sub-graph $N^{\prime}$ of $H^{\prime}$ that connects every relevant terminal pair, and is therefore a feasible solution to $I^{\prime}$. It is easy to see that $N^{\prime}$ has the same cost as $N$, as $N^{\prime}$ uses the oriented version of each edge of $N$. Conversely, every feasible solution $N^{\prime}$ for $I^{\prime}$ corresponds to a subgraph $N$ of $H$ that M-connects every relevant terminal pair. Therefore, $N$ is a feasible solution to $I^{\prime}$ with the same cost as $N^{\prime}$. This establishes an efficiently computable one-toone correspondence between feasible solutions to $I$ that are contained in $H$ and feasible solutions to $I^{\prime}$. Since there is an optimum solution to $I$ contained in $H$ [9], this is an approximation-preserving reduction from directional 3D-MMN to DSF.

By means of the above transformation of the Hanan grid into a digraph, we also obtain an approximation-preserving reduction from 3D-RSA to DST. We use this later in Section 4 to develop an approximation algorithm for 3D-MMN. Let $I$ be an instance of 3D-RSA given by a set $T$ of 
terminals with non-negative coordinates that are to be M-connected to the origin $o$. We construct an instance $I^{\prime}$ of DSF as above where $\{o\} \times T$ is the set of node pairs to be connected. Note that any feasible solution to $I^{\prime}$ is, without loss of generality, a tree. Hence, $I^{\prime}$ is an instance of DST with root $o$. All in all, we have an approximation-preserving reduction from 3D-RSA to DST.

\section{The $k$-Plane Case}

In this section we consider $3 \mathrm{D}-\mathrm{MMN}$, under the assumption that the set $T$ of terminals is contained in the union of $k \geq 2$ planes $E_{1}, \ldots, E_{k}$ that are parallel to the $x-y$ plane. Of course, this assumption always holds for some $k \leq n$. We present a $4(k-1)$-approximation algorithm, which outperforms our algorithm for the general case in Section 4 if $k \in o\left(n^{\varepsilon}\right)$.

Let $N_{\text {opt }}$ be some fixed minimum M-network for $T$, let $N_{\text {opt }}^{\text {hor }}$ be the set of all $x$-aligned and all $y$-aligned segments in $N_{\text {opt }}$, and let $N_{\text {opt }}^{\mathrm{ver}}$ be the set of all $z$-aligned segments in $N_{\text {opt }}$. Let OPT denote the weight of $N_{\text {opt }}$. Clearly, OPT does not depend on the specific choice of $N_{\text {opt }}$; the weights of $N_{\mathrm{opt}}^{\mathrm{hor}}$ and $N_{\mathrm{opt}}^{\mathrm{ver}}$, however, may depend on $N_{\mathrm{opt}}$. For $i \in\{1, \ldots, k\}$, let $T_{i}=T \cap E_{i}$ be the set of terminals in plane $E_{i}$. Further, let $T_{x y}$ be the projection of $T$ onto the $x-y$ plane.

Our algorithm consists of two phases. Phase I computes a set $N^{\text {hor }}$ of horizontal (that is, $x$ - and $y$-aligned) line segments, phase II computes a set $N^{\text {ver }}$ of vertical (that is, $z$-aligned) line segments. Finally, the algorithm returns the set $N=N^{\text {hor }} \cup N^{\text {ver }}$.

Phase I is simple; we compute a 2-approximate M-network $N_{x y}$ for $T_{x y}$ (using the algorithm of Guo et al. [10]) and project $N_{x y}$ onto each of the planes $E_{1}, \ldots, E_{k}$. Let $N^{\text {hor }}$ be the union of these projections. Note that $N^{\text {hor }}$ M-connects any pair of terminals that lie in the same plane.

Observation $2\left\|N^{\text {hor }}\right\| \leq 2 k\left\|N_{\mathrm{opt}}^{\text {hor }}\right\|$.

Proof. The projection of $N_{\mathrm{opt}}^{\mathrm{hor}}$ to the $x-y$ plane is an M-network for $T_{x y}$. Hence, $\left\|N_{x y}\right\| \leq 2 N_{\mathrm{opt}}^{\mathrm{hor}}$. Adding up over the $k$ planes yields the claim.

In Phase II, we construct a pillar network by computing a set $N^{\mathrm{ver}}$ of vertical line segments, so-called pillars, of total cost at most $4(k-1)\left\|N_{\mathrm{opt}}^{\mathrm{ver}}\right\|$. This yields an overall approximation factor of $4(k-1)$ since $\left\|N^{\text {hor }} \cup N^{\text {ver }}\right\| \leq 2 k\left\|N_{\text {opt }}^{\text {hor }}\right\|+4(k-1)\left\|N_{\text {opt }}^{\text {ver }}\right\| \leq 4(k-1)\left(\left\|N_{\text {opt }}^{\text {hor }}\right\|+\left\|N_{\text {opt }}^{\text {ver }}\right\|\right) \leq 4(k-1)$ OPT.

Below we describe Phase II of our algorithm for the directional subproblem that runs in direction north-east (NE) in the $x-y$ plane (with increasing $z$-coordinates). For this directional subproblem, we construct a pillar network $N_{\mathrm{dir}}^{\mathrm{ver}}$ of weight at most $(k-1)\left\|N_{\mathrm{opt}}^{\mathrm{ver}}\right\|$ that, together with $N^{\text {hor }}$, Mconnects all relevant pairs. We solve the analogous subproblems for the directions NW, SE, and SW in the same fashion. Then $N^{\mathrm{ver}}$ is the union of the four partial solutions and has weight at most $4(k-1)\left\|N_{\text {opt }}^{\mathrm{ver}}\right\|$, as desired.

Our directional subproblem is closely linked to the (directional) bichromatic rectangle piercing problem (BRP), which is defined as follows. Let $R$ and $B$ be sets of red and blue points in $\mathbb{R}^{2}$, respectively, and let $\mathcal{R}(R, B)$ denote the set of axis-aligned rectangles each of which is spanned by a red point in its SW-corner and a blue point in its NE-corner. Then the aim of BRP is to find a minimum-cardinality set $P \subset \mathbb{R}^{2}$ such that every rectangle in $\mathcal{R}(R, B)$ is pierced, that is, contains at least one point in $P$. The points in $P$ are called piercing points.

The problem dual to $\mathrm{BRP}$ is the (directional) bichromatic independent set of rectangles problem (BIS) where the goal is to find the maximum number of pairwise disjoint rectangles in $\mathcal{R}(R, B)$, given the sets $R$ and $B$. 
Recently, Soto and Telha [17] proved a beautiful min-max theorem saying that, for $\mathcal{R}(R, B)$, the minimum number of piercing points always equals the maximum number of independent rectangles. This enabled them to give efficient exact algorithms for BRP and BIS running in $\tilde{O}\left(n^{2.5}\right)$ worst-case time or $\tilde{O}\left(n^{\gamma}\right)$ expected time, where the $\tilde{O}$-notation ignores polylogarithmic factors, $\gamma<2.4$ is the exponent for fast matrix multiplication, and $n=|R|+|B|$ is the input size.

The details of Phase II appear, for $k=2$ planes, in Section 3.1, and, for $k>2$ planes, in Section 3.2. Algorithm 1 summarizes of our $k$-planes algorithm.

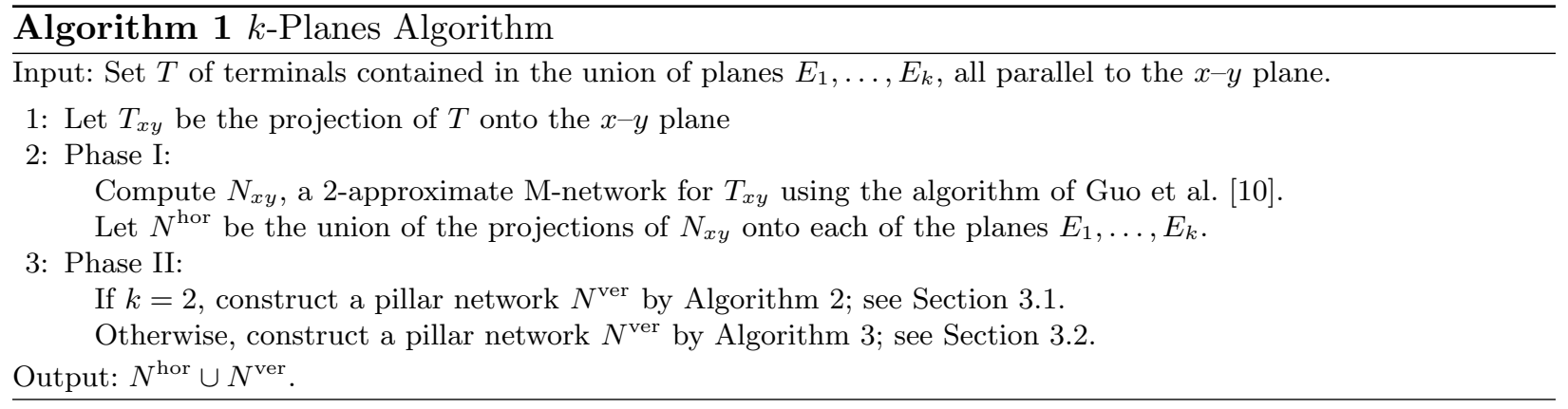

\subsection{Pillar Network for Two Planes}

Our phase-II algorithm for two planes is very simple. We sketch it first in order to provide some intuition for the $k$-planes case. Let the terminals in $T_{1}$ be red and those in $T_{2}$ be blue. Ignore the $z$-coordinates of the terminals. Then the relevant red-blue point pairs span exactly the rectangles in $\mathcal{R}\left(T_{1}, T_{2}\right)$, which we call relevant, too.

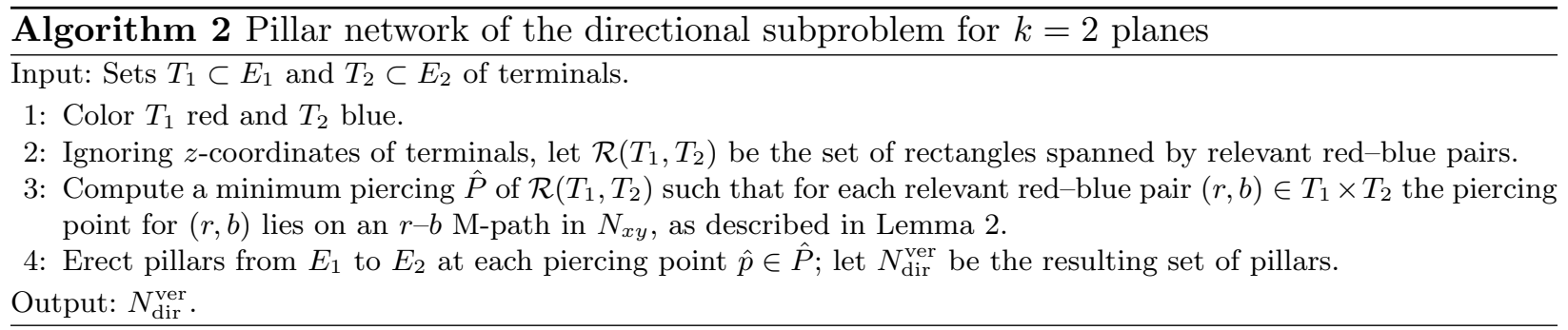

Our algorithm (Algorithm 2 consists of two steps. First, we compute a minimum piercing $P$ of $\mathcal{R}\left(T_{1}, T_{2}\right)$ using the algorithm of Soto and Telha [17]. Second, we move each piercing point $p \in P$ to a new position $\hat{p}$ - a nearby junction of $N_{x y}$-and erect, at $\hat{p}$, a pillar connecting the two planes.

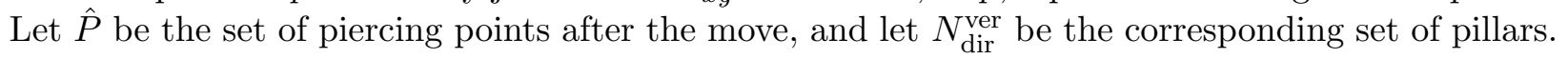

Lemma 1. It holds that $\left\|N_{\text {dir }}^{\mathrm{ver}}\right\| \leq\left\|N_{\mathrm{opt}}^{\mathrm{ver}}\right\|$.

Proof. It is easy to see that $|\hat{P}|=|P|$. Integrating over the distance $d$ of the two planes yields $\left\|N_{\text {dir }}^{\text {ver }}\right\|=|\hat{P}| \cdot d=|P| \cdot d \leq\left\|N_{\text {opt }}^{\text {ver }}\right\|$. The last inequality is due to the fact that $P$ is a minimum piercing of $\mathcal{R}\left(T_{1}, T_{2}\right)$ and that the pillars in $N_{\text {opt }}^{\text {ver }}$ pierce $\mathcal{R}\left(T_{1}, T_{2}\right)$-otherwise $N_{\text {opt }}$ would not be feasible. 
Now we turn to feasibility. We first detail how we move each piercing point $p$ to its new position $\hat{p}$. For the sake of brevity, we identify terminals with their projections to the $x-y$ plane. Our description assumes that we have at our disposal some network $M$ (such as $N_{x y}$ ) connecting the relevant pairs in $T_{x y}$.

For a piercing point $p \in P$, let $A_{p}$ be the intersection of the relevant rectangles pierced by $p$; see Fig. 3. Clearly, $p \in A_{p}$. Note that the bottom and left sides of $A_{p}$ are determined by terminals $t_{\mathrm{W}}$ and $t_{\mathrm{S}}$ to the west and south of $A_{p}$, respectively. Symmetrically, the top and right sides of $A_{p}$ are determined by terminals $t_{\mathrm{E}}$ and $t_{\mathrm{N}}$ to the east and north of $A_{p}$, respectively. Terminals $t_{\mathrm{W}}$ and $t_{\mathrm{S}}$ may coincide, and so may $t_{\mathrm{E}}$ and $t_{\mathrm{N}}$. It is easy to see that the network $M$ contains an M-path $\pi_{\mathrm{SN}}$ connecting $t_{\mathrm{S}}$ and $t_{\mathrm{N}}$ and an M-path $\pi_{\mathrm{WE}}$ connecting $t_{\mathrm{W}}$ and $t_{\mathrm{E}}$. The path $\pi_{\mathrm{SN}}$ goes through the bottom and top sides of $A_{p}$ and $\pi_{\mathrm{WE}}$ goes through the left and right sides. Hence, the two paths intersect in a point $\hat{p} \in A_{p}$. This is where we move the original piercing point $p$.

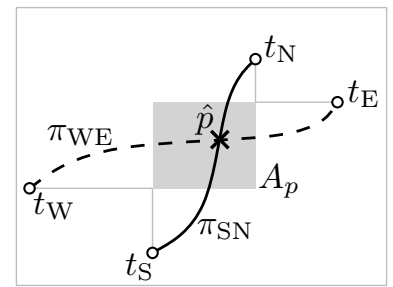

Fig. 3: Paths $\pi_{\mathrm{SN}}$ and $\pi_{\mathrm{WE}}$ meet in a point $\hat{p}$ in $A_{p}$.

Since $\hat{p} \in A_{p}$, the point $\hat{p}$ pierces the same relevant rectangles as $p$, and the set $\hat{P}=\{\hat{p} \mid p \in P\}$ is a (minimum) piercing for the set of relevant rectangles.

Lemma 2. Let $\mathcal{R}(R, B)$ be an instance of BRP and let $M$ be a network that $M$-connects every relevant red-blue point pair. Then we can efficiently compute a minimum piercing of $\mathcal{R}(R, B)$ such that $M$ contains, for every relevant red-blue point pair $(r, b)$ in $R \times B$, an $r-b$ M-path that contains a piercing point.

Proof. We use the algorithm of Soto and Telha [17] to compute a minimum piercing $P$ of $\mathcal{R}(R, B)$. Then, as we have seen above, $\hat{P}$ is a minimum piercing of $\mathcal{R}(R, B)$, too. Now let $(r, b)$ be a relevant red-blue pair in $R \times B$, and let $p \in P$ be a point that pierces $R(r, b)$. Clearly, $\hat{p}$ pierces $R(r, b)$, too. As we have observed before, both $p$ and $\hat{p}$ lie in $A_{p}$.

Since $(r, b)$ is a relevant pair, $r$ lies to the SW of $A_{p}$ and $b$ to the NE; see Fig. 4 a. We prove that $M$ contains an $r-\hat{p}$ M-path; a symmetric argument proves that $M$ also contains a $\hat{p}-b$ M-path. Concatenating these two M-paths yields the desired $r-b$ M-path since $r$ lies to the SW of $\hat{p}$ and $\hat{p}$ lies to the SW of $b$. Recall that $\hat{p}$ lies on the intersection of the $t_{\mathrm{W}}-t_{\mathrm{E}}$ M-path $\pi_{\mathrm{WE}}$ and the $t_{\mathrm{S}}-t_{\mathrm{N}}$ M-path $\pi_{\mathrm{SN}}$, where $t_{\mathrm{W}}, t_{\mathrm{E}}, t_{\mathrm{S}}, t_{\mathrm{N}}$ are the terminals that determine the extensions of $A_{p}$; see Fig. 3 . To show that $M$ M-connects $r$ and $\hat{p}$, we consider two cases.

Case I: $r \in R\left(t_{\mathrm{W}}, t_{\mathrm{S}}\right)$; see Fig. $4 \mathrm{a}$. According to our assumption, $M$ contains some $r-b \mathrm{M}$-path $\pi$. Then $\pi$ must intersect $\pi_{\mathrm{WE}}$ or $\pi_{\mathrm{SN}}$ at some point $x$ to the $\mathrm{SW}$ of $\hat{p}$. Thus, we can go, in a monotone fashion, along $\pi$ from $r$ to $x$ and then along $\pi_{\mathrm{WE}}$ or $\pi_{\mathrm{SN}}$ from $x$ to $\hat{p}$. This is the desired $r-\hat{p}$ M-path.

Case II: $r$ lies to the SW of $t_{\mathrm{W}}$ or $t_{\mathrm{S}}$; see Fig. $4 \mathrm{~b}$. In this case $M$ contains M-paths from $r$ to $t_{\mathrm{W}}$ and to $t_{\mathrm{S}}$. If $r$ lies to the $\mathrm{SW}$ of $t_{\mathrm{W}}$, we can go, again in a monotone fashion, from $r$ to $t_{\mathrm{W}}$ and 


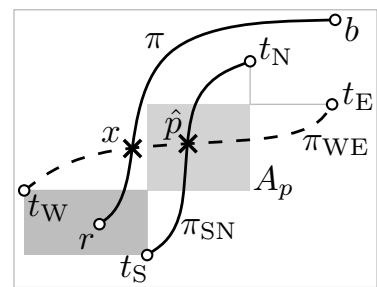

(a) Case I: we can go from $r$ via $x$ to $\hat{p}$.

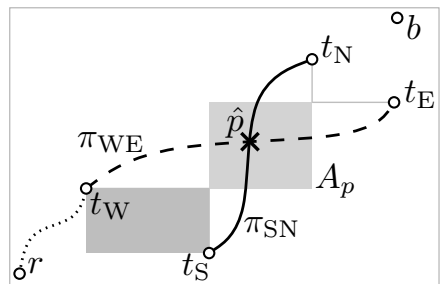

(b) Case II: we can go from $r$ via $t_{\mathrm{W}}$ or $t_{\mathrm{S}}$ to $\hat{p}$.

Fig. 4: Sketches for the proof of Lemma 2

then along $\pi_{\mathrm{WE}}$ from $t_{\mathrm{W}}$ to $\hat{p}$. Otherwise, if $r$ lies to the SW of $t_{\mathrm{S}}$, we can go from $r$ to $t_{\mathrm{S}}$ and then on $\pi_{\mathrm{SN}}$ from $t_{\mathrm{S}}$ to $\hat{p}$.

Since these are the only two possibilities, this concludes the proof.

Lemmas 1 and 2 (with $R=T_{1}, B=T_{2}$, and $M=N_{x y}$ ) yield the following.

Theorem 2. We can efficiently compute a 4-approximation for the 2-plane case.

\subsection{Pillar Network for $k$ Planes}

Now we show how our phase-II algorithm generalizes to $k$ planes. As in the 2-planes case, we restrict ourselves to the directional subproblem and construct a pillar network $N_{\text {dir }}^{\mathrm{ver}}$ of weight at most $(k-1)\left\|N_{\mathrm{opt}}^{\mathrm{ver}}\right\|$. As we have argued at the beginning of Section 3, this suffices to prove Theorem 3 .

Theorem 3. There exists a 4(k-1)-approximation algorithm for 3D-MMN where the terminals lie in the union of $k \geq 2$ planes parallel to the $x-y$ plane.

Our pillar-placement algorithm (Algorithm 3) is as follows. Let $i \in\{1, \ldots, k-1\}$. We construct an instance $\mathcal{I}_{i}$ of BRP where we two-color $T_{x y}$ such that each point corresponding to a terminal of some plane $E_{j}$ with $j \leq i$ is colored red and each point corresponding to a terminal of some plane $E_{j^{\prime}}$ with $j^{\prime} \geq i+1$ is colored blue. For $\mathcal{I}_{i}$, we compute a minimum piercing $\hat{P}_{i}$ according to Lemma 2 with $M=N_{x y}$. In other words, for any relevant pair $\left(t_{j}, t_{j^{\prime}}\right)$, there is some M-path in $N_{x y}$ that contains a piercing point of $\hat{P}_{i}$. We choose $i^{\star} \in\{1, \ldots, k-1\}$ such that $\hat{P}_{i^{\star}}$ has minimum cardinality. This is crucial for our analysis. At the piercing points of $\hat{P}_{i^{\star}}$, we erect pillars spanning all planes $E_{1}, \ldots, E_{k}$. Let $\hat{N}_{i^{\star}}$ be the set of these pillars. We now show that $\hat{N}_{i^{\star}}$, along with $N^{\text {hor }}$, creates a feasible network for any relevant terminal pair $\left(t_{j}, t_{j}^{\prime}\right)$ such that $j \leq i^{\star}$ and $j^{\prime} \geq i^{\star}+1$.

Lemma 3. The network $N^{\text {hor }} \cup \hat{N}_{i^{\star}}$ M-connects any relevant terminal pair in $T_{j} \times T_{j^{\prime}}$ with $j \leq i^{\star}$ and $j^{\prime} \geq i^{\star}+1$.

Proof. Consider a pair $\left(t_{j}, t_{j^{\prime}}\right)$ in $T_{j} \times T_{j^{\prime}}$ as in the statement. We construct an M-path from $t_{j}$ to $t_{j^{\prime}}$ as follows. We know that there exists an M-path $\pi$ that connects the projections of $t_{j}$ and $t_{j^{\prime}}$ in $N_{x y}$ and contains a piercing point $p$ of $\hat{P}_{i^{\star}}$. Therefore, we can start at $t_{j}$ and follow the projection of $\pi$ onto plane $E_{j}$ until we arrive at $p$. Then we use the corresponding pillar in $\hat{N}_{i^{\star}}$ to reach the plane $E_{j^{\prime}}$, where we follow the projection of $\pi$ (onto that plane) until we reach $t_{j^{\prime}}$. 


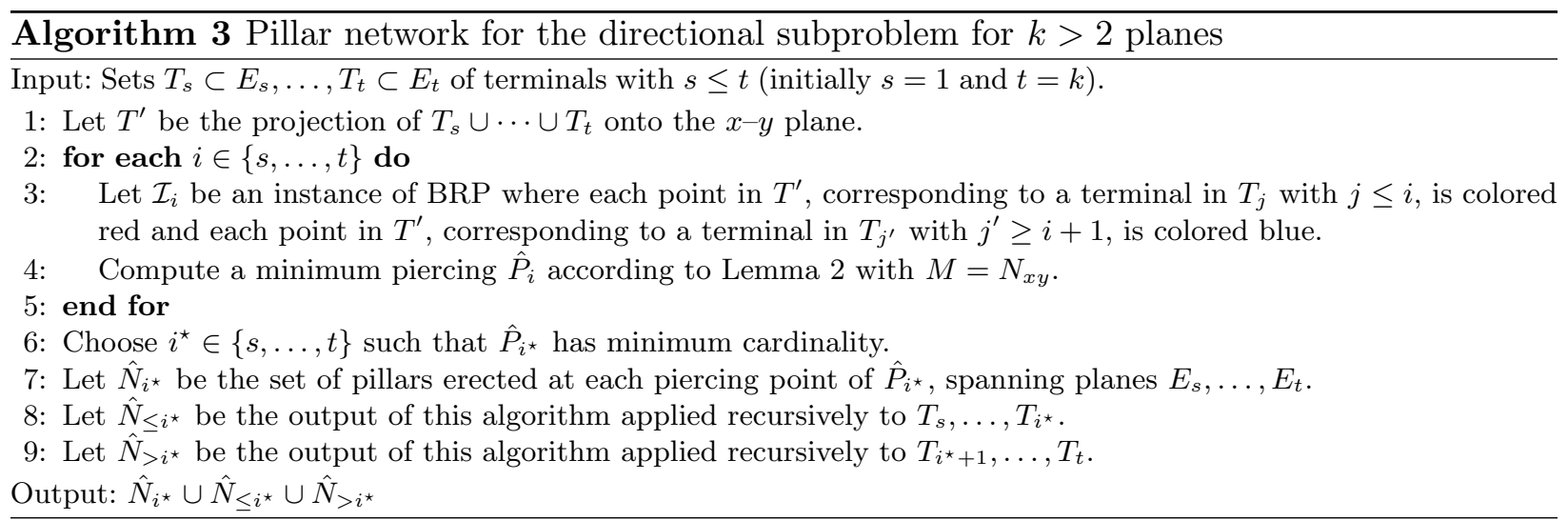

In order to also M-connect relevant terminal pairs in $T_{j} \times T_{j^{\prime}}$, where either $\left(j \leq i^{\star}\right.$ and $\left.j^{\prime} \leq i^{\star}\right)$ or $\left(j \geq i^{\star}+1\right.$ and $\left.j^{\prime} \geq i^{\star}+1\right)$, we simply apply the pillar-placement algorithm recursively to the sets $T_{1}, \ldots, T_{i^{\star}}$ and $T_{i^{\star}+1}, \ldots, T_{k}$. This yields the desired pillar network $N_{\text {dir }}^{\text {ver }}$. By Lemma $3, N_{\text {dir }}^{\text {ver }} \cup N^{\text {hor }}$ is feasible. Next, we bound $\left\|\hat{N}_{i^{\star}}\right\|$.

Lemma 4. Let $M$ be an arbitrary directional Manhattan network for $T$, and let $M^{\mathrm{ver}}$ be the set of vertical segments in $M$. Then the pillar network $\hat{N}_{i^{\star}}$ has weight at most $\left\|M^{\mathrm{ver}}\right\|$.

Proof. Without loss of generality, we assume that $M$ is a subnetwork of the Hanan grid [9]. We may also assume that any segment of $M^{\text {ver }}$ spans only consecutive planes. For $1 \leq i \leq j \leq k$, let $M_{i, j}$ denote the subnetwork of $M^{\text {ver }}$ lying between planes $E_{i}$ and $E_{j}$. Let $d_{i, j}$ be the vertical distance between planes $E_{i}$ and $E_{j}$.

We start with the observation that, for any $j=1, \ldots, k-1$, the network $M_{j, j+1}$ is a set of pillars that forms a valid piercing of the piercing instance $\mathcal{I}_{j}$ (defined right after Theorem 3 ). Hence, $\left|M_{j, j+1}\right| \geq\left|\hat{P}_{j}\right| \geq\left|\hat{P}_{i^{\star}}\right|$, which implies the claim of the lemma as follows:

$$
\left\|M^{\mathrm{ver}}\right\|=\sum_{j=1}^{k-1}\left\|M_{j, j+1}\right\|=\sum_{j=1}^{k-1}\left|M_{j, j+1}\right| \cdot d_{j, j+1} \geq \sum_{j=1}^{k-1}\left|P_{i^{\star}}\right| \cdot d_{j, j+1}=\left|P_{i^{\star}}\right| \cdot d_{1, k}=\left\|P_{i^{\star}}\right\| .
$$

It is crucial for our construction that the pillars constructed recursively span either $E_{1}, \ldots, E_{i^{\star}}$ or $E_{i^{\star}+1}, \ldots, E_{k}$, but not all planes. For $1 \leq j \leq j^{\prime} \leq k$, let weight $z\left(j, j^{\prime}\right)$ denote the weight of the vertical part of the network produced by the above pillar-placement algorithm, when applied to planes $E_{j}, \ldots, E_{j^{\prime}}$ recursively. For technical reasons we set weight $z(j, j)=0$. Now assume that $j<j^{\prime}$ and that the algorithm makes the partition at plane $E_{i^{\prime}}$ with $j \leq i^{\prime}<j^{\prime}$ when planes $E_{j}, \ldots, E_{j^{\prime}}$ are processed. By means of Lemma 4 , we derive the recursion

$$
\text { weight }_{z}\left(j, j^{\prime}\right) \leq\left\|M_{j, j^{\prime}}\right\|+\text { weight }_{z}\left(j, i^{\prime}\right)+\operatorname{weight}_{z}\left(i^{\prime}+1, j^{\prime}\right) \text {, }
$$

which holds for any M-network $M$ for $T$. We now claim that

$$
\text { weight }_{z}\left(j, j^{\prime}\right) \leq\left(j^{\prime}-j\right)\left\|M_{j, j^{\prime}}\right\| \text {. }
$$


Our proof is by induction on the number of planes processed by the algorithm. By the inductive hypothesis, we have that weight $z\left(j, i^{\prime}\right) \leq\left(i^{\prime}-j\right)\left\|M_{j, i^{\prime}}\right\|$ and weight $_{z}\left(i^{\prime}+1, j^{\prime}\right) \leq\left(j^{\prime}-i^{\prime}-\right.$ 1) $\left\|M_{i^{\prime}+1, j^{\prime}}\right\|$. We plug these expressions into the recursion 11. Since $\left\|M_{j, i^{\prime}}\right\|+\left\|M_{i^{\prime}+1, j^{\prime}}\right\| \leq\left\|M_{j, j^{\prime}}\right\|$ and weight $z(l, l)=0$ for any $l \in\{1, \ldots, k\}$, the claim follows.

We conclude that the weight of the solution produced by the algorithm, when applied to all planes $E_{1}, \ldots, E_{k}$, is bounded by weight $z(1, k) \leq(k-1)\left\|M_{1, k}\right\|=(k-1)\left\|M^{\text {ver }}\right\|$. This completes the proof of Theorem 3 .

\section{The General Case}

In this section, we present an approximation algorithm, which we call the grid algorithm, for the general 3D-MMN problem. Our main result is the following.

Theorem 4. For any $\varepsilon>0$, there exists an $O\left(n^{\varepsilon}\right)$-approximation algorithm for $3 D$-MMN that, given a set of $n$ terminals, runs in $n^{O(1 / \varepsilon)}$ time.

This result is better than the one in the previous section if the given set of terminals is distributed over $\omega\left(n^{\varepsilon}\right)$ horizontal planes. Moreover, the approach in this section extends to higher dimensions; see appendix.

For technical reasons, we assume that the terminals are in general position, that is, any two terminals differ in all three coordinates. By Observation 1 it suffices to describe and analyze the algorithm for the directional subproblem.

\subsection{The 3D Grid Algorithm}

We begin the description with a high-level summary. To solve the directional subproblem, we place a 3D grid that partitions the instance into a constant number of cuboids; see Fig. 5a. Cuboids that differ in only two coordinates form slabs. We connect terminals from different slabs by M-connecting each terminal to the corners of its cuboid and by using the edges of the grid to connect the corners. We connect terminals from the same slab by recursively applying our algorithm to the slabs.

Step 1: Partitioning into cuboids and slabs. Consider the bounding cuboid $C$ of $T$ and set $c=3^{1 / \varepsilon}$. Partition $C$ by $3(c-1)$ separating planes into $c \times c \times c$ axis-aligned subcuboids $C_{i j k}$ with $i, j, k \in$ $\{1, \ldots, c\}$. The indices are such that larger indices mean larger coordinates. Place the separating planes such that the number of terminals between two consecutive planes is at most $n / c$. This can be accomplished by executing a simple plane-sweep for each direction $x, y, z$, and by placing separating planes after every $n / c$ terminals. Here we exploit our general-position assumption. The edges of the resulting subcuboids - except the edges on the boundary of $C$, which we do not need -induce a three-dimensional grid $\mathcal{G}$ of axis-aligned line segments. We insert $\mathcal{G}$ into the solution.

For each $i \in\{1, \ldots, c\}$, define the $x$-aligned slab, $C_{i}^{x}$, to be the union of all cuboids $C_{i j k}$ with $j, k \in\{1, \ldots, c\}$. Define $y$-aligned and $z$-aligned slabs $C_{j}^{y}, C_{k}^{z}$ analogously; see Fig. $5 \mathrm{~b}$.

Step 2: Add M-paths between different slabs. Consider two cuboids $C_{i j k}$ and $C_{i^{\prime} j^{\prime} k^{\prime}}$ with $i<i^{\prime}$, $j<j^{\prime}$, and $k<k^{\prime}$. Any terminal pair $\left(t, t^{\prime}\right) \in C_{i j k} \times C_{i^{\prime} j^{\prime} k^{\prime}}$ can be M-connected using the edges of $\mathcal{G}$ as long as $t$ and $t^{\prime}$ are connected to the appropriate corners of their cuboids; see Fig. $5 \mathrm{c}$. To this end, we use the following patching procedure.

Call a cuboid $C_{i j k}$ relevant if there is a non-empty cuboid $C_{i^{\prime} j^{\prime} k^{\prime}}$ with $i<i^{\prime}, j<j^{\prime}$, and $k<k^{\prime}$. For each relevant cuboid $C_{i j k}$, let $\hat{p}_{i j k}$ denote a corner that is dominated by all terminals inside $C_{i j k}$. 


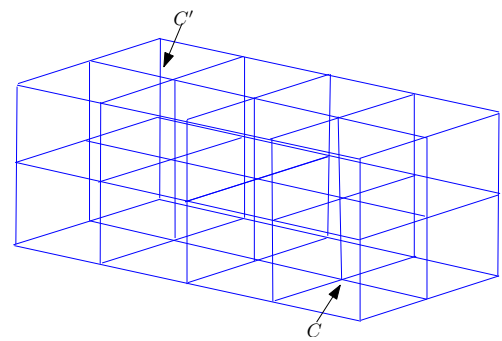

(a) Example of a grid with two cuboids from different slabs.

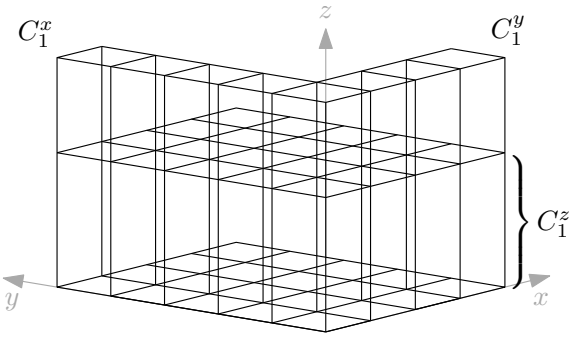

(b) Examples of $x-, y$-, and $z$-aligned slabs.

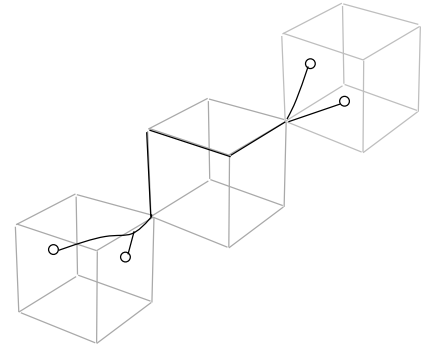

(c) M-paths using patching and grid edges.

Fig. 5: Illustrations for the grid algorithm: cuboids, slabs, and patches.

We define up-patching $C_{i j k}$ to mean M-connecting every terminal in $C_{i j k}$ to $\hat{p}_{i j k}$. We up-patch $C_{i j k}$ by solving (approximately) an instance of 3D-RSA with the terminals in $C_{i j k}$ as points and $\hat{p}_{i j k}$ as origin. We define down-patching analogously; cuboid $C_{i j k}$ is relevant if there is a non-empty cuboid $C_{i^{\prime} j^{\prime} k^{\prime}}$ with $i>i^{\prime}, j>j^{\prime}, k>k^{\prime}$; we let $\check{p}_{i j k}$ be the corner that dominates all terminals in $C_{i j k}$.

We complete this step by inserting the up-patches and the down-patches of all relevant cuboids into the solution.

Step 3: Add M-paths within slabs. To M-connect relevant terminal pairs that lie in the same slab, we apply the grid algorithm (steps 1-3) recursively to each slab $C_{i}^{x}, C_{j}^{y}$, and $C_{k}^{z}$ with $i, j, k \in\{1, \ldots, c\}$.

\subsection{Analysis}

We first show that the output of the algorithm presented in Section 4.1 is feasible, then we establish its approximation ratio of $O\left(n^{\varepsilon}\right)$ and its running time of $n^{O(1 / \varepsilon)}$ for any $\varepsilon>0$. In this section, OPT denotes the weight of a minimum M-network (not the cost of an optimal solution to the directional subproblem).

Lemma 5 (Feasibility). The grid algorithm M-connects all relevant terminal pairs.

Proof. Let $\left(t, t^{\prime}\right)$ be a relevant terminal pair. First, suppose that $t$ and $t^{\prime}$ lie in cuboids of different slabs. Thus, there are $i<i^{\prime}, j<j^{\prime}, k<k^{\prime}$ such that $t \in C_{i j k}$ and $t^{\prime} \in C_{i^{\prime} j^{\prime} k^{\prime}}$. Furthermore, $C_{i j k}$ and $C_{i^{\prime} j^{\prime} k^{\prime}}$ are relevant for up- and down-patching, respectively. When up-patching, we solve an instance of RSA connecting all terminals in $C_{i j k}$ to $\hat{p}_{i j k}$. Similarly, down-patching M-connects $t^{\prime}$ to $\check{p}_{i^{\prime} j^{\prime} k^{\prime}}$. The claim follows as $\mathcal{G}$ M-connects $\hat{p}_{i j k}$ and $\check{p}_{i^{\prime} j^{\prime} k^{\prime}}$.

Now, suppose that $t$ and $t^{\prime}$ lie in the same slab. As the algorithm is applied recursively to each slab, there will be a recursion step where $t$ and $t^{\prime}$ lie in cuboids in different slabs. Here, we need our general-position assumption. Applying the argument above to that particular recursive step completes the proof.

Approximation ratio. Next, we turn to the performance of our algorithm. Let $r(n)$ be its approximation ratio, where $n$ is the number of terminals in $T$. The total weight of the output is the sum of $\|\mathcal{G}\|$, the cost of patching, and the cost for the recursive treatment of the slabs. We analyze each of the three costs separately. 
The grid $\mathcal{G}$ consists of all edges induced by the $c^{3}$ subcuboids except the edges on the boundary of $C$. Let $\ell$ denote the length of the longest side of $C$. The weight of $\mathcal{G}$ is at most $3(c-1)^{2} \ell$, which is bounded by $3 c^{2} \mathrm{OPT}$ as $\ell \leq \mathrm{OPT}$.

Let $r_{\text {patch }}(n)$ denote the cost of patching all relevant cuboids in step 2. Lemma 6 (given below) proves that $r_{\text {patch }}(n)=O\left(n^{\varepsilon}\right) \mathrm{OPT}$.

Now consider the recursive application of the algorithm to all slabs. Recall that $N_{\text {opt }}$ is a fixed minimum M-network for $T$. For $i \in 1, \ldots, c$, let $\mathrm{OPT}_{i}^{x}$ be the optimum cost for M-connecting all (not only relevant) terminal pairs in slab $C_{i}^{x}$. Define $\mathrm{OPT}_{i}^{y}$ and $\mathrm{OPT}_{i}^{z}$ analogously.

Slightly abusing of notation, we write $N_{\text {opt }} \cap C_{i}^{x}$ for the set $\left\{s \cap C_{i}^{x} \mid s \in N_{\text {opt }}\right\}$ of line segments of $N_{\text {opt }}$ intersected with slab $C_{i}^{x}$. Observe that $N_{\text {opt }} \cap C_{i}^{x}$ forms a feasible solution for $C_{i}^{x}$. Thus, $\mathrm{OPT}_{i}^{x} \leq\left\|N_{\text {opt }} \cap C_{i}^{x}\right\|$. By construction, any slab contains at most $n / c$ terminals. Hence, the total cost of the solutions for slabs $C_{1}^{x}, \ldots, C_{c}^{x}$ is at most

$$
\sum_{i=1}^{c} r\left(\frac{n}{c}\right) \mathrm{OPT}_{i}^{x} \leq r\left(\frac{n}{c}\right) \sum_{i=1}^{c}\left\|N_{\mathrm{opt}} \cap C_{i}^{x}\right\| \leq r\left(\frac{n}{c}\right) \text { OPT }
$$

Clearly, the solutions for the $y$ - and $z$-slabs have the same bound.

Summing up all three types of costs, we obtain the recursive equation

$$
r(n) \mathrm{OPT} \leq 3 c^{2} \mathrm{OPT}+r_{\mathrm{patch}}(n) \mathrm{OPT}+3 r\left(\frac{n}{c}\right) \mathrm{OPT} .
$$

Hence, $r(n)=O\left(n^{\max \left\{\varepsilon, \log _{c} 3\right\}}\right)$. Plugging in $c=3^{1 / \varepsilon}$ yields $r(n)=O\left(n^{\varepsilon}\right)$, which proves the approximation ratio claimed in Theorem 4 ,

Lemma 6. Patching all relevant cuboids costs $r_{\text {patch }}(n) \in O\left(n^{\varepsilon}\right) \mathrm{OPT}$.

Proof. First note that it suffices to consider up-patching; the down-patching case can be argued analogously.

Lemma 7 shows the existence of a near-optimal M-network that up-patches all relevant cuboids. Lemma 8 shows that by reducing the patching problem to 3D-RSA, we can find such a network of cost $O(\rho) \mathrm{OPT}$, where $\rho$ is the approximation factor of 3D-RSA.

We argued in Section 2.3 that there exists an approximation-preserving reduction from 3D-RSA to DST. DST, in turn, admits an $O\left(n^{\varepsilon}\right)$-approximation for any $\varepsilon>0$ [4]. Hence, the cost of uppatching is indeed bounded by $O\left(n^{\varepsilon}\right) \mathrm{OPT}$.

We now turn to the two lemmas that we just used in the proof of Lemma 6. For our analysis, we need the network $N^{\prime}$ that is the union of $\mathcal{G}$ with $N_{\text {opt }}$ and the projections of $N_{\text {opt }}$ onto every separating plane of $\mathcal{G}$. Since there are $3(c-1)$ separating planes and, as we have seen above, $\|\mathcal{G}\| \leq 3 c^{2} \mathrm{OPT}$, it holds that $\left\|N^{\prime}\right\| \leq 3\left(c^{2}+c\right) \mathrm{OPT}=O(\mathrm{OPT})$.

Lemma 7. There exists an M-network of total cost at most $3\left(c^{2}+c\right) \mathrm{OPT}$ that up-patches all relevant cuboids.

Proof. We claim that $N^{\prime}$ up-patches all relevant cuboids. To this end, let $t \in C_{i j k}$ and let $t^{\prime} \in C_{i^{\prime} j^{\prime} k^{\prime}}$ with $i<i^{\prime}, j<j^{\prime}, k<k^{\prime}$. Follow the M-path connecting $t$ and $t^{\prime}$, starting from $t$. This path must leave $C_{i j k}$ at a certain point $\bar{p}$, which lies on some face $F$ of $C_{i j k}$. Face $F$, in turn, lies on some separating plane $S$ of the grid $\mathcal{G}$. From now on follow the projection of the M-path from $\bar{p}$ to $t^{\prime}$ on 
plane $S$. This projected path must leave the face $F$, since $t^{\prime}$ lies in $C_{i^{\prime} j^{\prime} k^{\prime}}$ with $i<i^{\prime}, j<j^{\prime}, k<k^{\prime}$, and the projection of $t^{\prime}$ onto $S$ must therefore lie outside of $F$. Moreover, the point $\bar{p}^{\prime}$ where this path leaves $F$ must lie on an edge of $C_{i j k}$ incident to $p_{i j k}$. Hence, we obtain a $t-p_{i j k}$ M-path by going from $t$ to $\bar{p}$, from $\bar{p}$ to $\bar{p}^{\prime}$ and then from $\bar{p}^{\prime}$ to $p_{i j k}$.

Lemma 8. Given a number $\rho \geq 1$ and an efficient $\rho$-approximation of 3D-RSA, we can efficiently up-patch all relevant cuboids at cost no more than $12\left(c^{2}+c\right) \rho \mathrm{OPT}$.

Proof. In Lemma 7, we showed the existence of a network $N^{\prime}$ that up-patches all relevant cuboids at low cost. Now consider an arbitrary relevant cuboid $C_{i j k}$. Clearly $N^{\prime} \cap C_{i j k}$ up-patches $C_{i j k}$. Hence $\mathrm{OPT}_{i j k}^{\mathrm{up}} \leq\left\|N^{\prime} \cap C_{i j k}\right\|$, where $\mathrm{OPT}_{i j k}^{\mathrm{up}}$ denotes the cost of a minimum up-patching of $C_{i j k}$. The problem of optimally up-patching $C_{i j k}$ is just an instance $I_{i j k}$ of 3D-RSA in which all terminals in $C_{i j k}$ have to be connected by an M-path to $\hat{p}_{i j k}$. Applying the factor- $\rho$ approximation algorithm for $3 \mathrm{D}-\mathrm{RSA}$ to each instance $I_{i j k}$ with $C_{i j k}$ relevant, we patch at total cost at most

$$
\rho \sum_{C_{i j k} \text { relevant }} \mathrm{OPT}_{i j k}^{\mathrm{up}} \leq \rho \sum_{C_{i j k} \text { relevant }}\left\|N^{\prime} \cap C_{i j k}\right\| \leq 4 \rho\left\|N^{\prime}\right\|
$$

The last inequality follows from the fact that each edge of $N^{\prime}$ occurs in at most four cuboids. The lemma follows since $\left\|N^{\prime}\right\| \leq 3\left(c^{2}+c\right)$ OPT.

Running time. Finally, we analyze the running time. Let $T(n)$ denote the running time of the algorithm applied to a set of $n$ terminals. The running time is dominated by patching and the recursive slab treatment. Using the DST algorithm of Charikar et al. [4], patching cuboid $C_{i}$ requires

time $n_{i}^{O(1 / \varepsilon)}$, where $n_{i}$ is the number of terminals in $C_{i}$. As each cuboid is patched at most twice and there are $c^{3}$ cuboids, patching takes $O\left(c^{3}\right) n^{O(1 / \varepsilon)}=n^{O(1 / \varepsilon)}$ time. The algorithm is applied recursively to $3 c$ slabs. This yields the recurrence $T(n)=3 c T(n / c)+n^{O(1 / \varepsilon)}$, which leads to the claimed running time.

This completes the proof of Theorem 4.

\section{Open Problems}

We have presented, for any $\varepsilon>0$, a grid-based $O\left(n^{\varepsilon}\right)$-approximation algorithm for $d \mathrm{D}$-MMN. This is a significant improvement over the ratio of $O\left(n^{4 / 5+\varepsilon}\right)$ which is achieved by reducing the problem to DSF. For 3D, we have described a $4(k-1)$-approximation algorithm for the case when the terminals lie on $k \geq 2$ horizontal planes. This outperforms our grid-based algorithm when $k \in o\left(n^{\varepsilon}\right)$. Whereas 2D-MMN admits a 2-approximation [5]10]15], it remains open whether $O(1)$ or $O(\log n)$-approximation algorithms exist for higher dimensions.

Our $O\left(n^{\varepsilon}\right)$-approximation algorithm for $d \mathrm{D}$-MMN solves instances of $d \mathrm{D}$-RSA for the subproblem of patching. We conjecture that $d \mathrm{D}$-RSA admits better approximation ratios. While this is an interesting open question, a positive result would still not be enough to improve our approximation ratio, which is dominated by the cost of finding M-paths inside slabs.

The complexity of the undirectional bichromatic rectangle piercing problem (see Section 3) is still unknown. Currently, the best approximation has a ratio of 4 , which is (trivially) implied by the result of Soto and Telha [17]. Any progress would immediately improve the approximation ratio of our algorithm for the $k$-plane case of 3D-MMN (for any $k>2$ ). 
Acknowledgments. This work was started at the 2009 Bertinoro Workshop on Graph Drawing. We thank the organizers Beppe Liotta and Walter Didimo for creating an inspiring atmosphere. We also thank Steve Wismath, Henk Meijer, Jan Kratochvíl, and Pankaj Agarwal for discussions. We are indebted to Stefan Felsner for pointing us to Soto and Telha's work [17].

\section{References}

1. Arora, S.: Approximation schemes for NP-hard geometric optimization problems: A survey. Math. Program. 97(1-2), 43-69 (2003)

2. Arya, S., Das, G., Mount, D.M., Salowe, J.S., Smid, M.: Euclidean spanners: Short, thin, and lanky. In: Proc. 27th Annu. ACM Sympos. Theory Comput. (STOC'95), pp. 489-498 (1995). URL http://dx.doi.org/10.1145/ 225058.225191

3. Benkert, M., Wolff, A., Widmann, F., Shirabe, T.: The minimum Manhattan network problem: Approximations and exact solutions. Comput. Geom. Theory Appl. 35(3), 188-208 (2006). URL http://dx.doi.org/10.1016/ j.comgeo.2005.09.004

4. Charikar, M., Chekuri, C., Cheung, T.Y., Dai, Z., Goel, A., Guha, S., Li, M.: Approximation algorithms for directed Steiner problems. In: Proc. 9th ACM-SIAM Sympos. Discrete Algorithms (SODA'98), pp. 192-200 (1998)

5. Chepoi, V., Nouioua, K., Vaxès, Y.: A rounding algorithm for approximating minimum Manhattan networks. Theor. Comput. Sci. 390(1), 56-69 (2008). URL http://dx.doi.org/10.1016/j.tcs.2007.10.013

6. Chin, F., Guo, Z., Sun, H.: Minimum Manhattan network is NP-complete. Discrete Comput. Geom. 45, 701-722 (2011). URL http://dx.doi.org/10.1007/s00454-011-9342-z

7. Feldman, M., Kortsarz, G., Nutov, Z.: Improved approximating algorithms for directed Steiner forest. In: Proc. 20th ACM-SIAM Sympos. Discrete Algorithms (SODA'09), pp. 922-931 (2009)

8. Fuchs, B., Schulze, A.: A simple 3-approximation of minimum Manhattan networks. Tech. Rep. 570, Zentrum für Angewandte Informatik Köln (2008). URL http://e-archive.informatik.uni-koeln.de/570

9. Gudmundsson, J., Levcopoulos, C., Narasimhan, G.: Approximating a minimum Manhattan network. Nordic J. Comput. 8, 219-232 (2001)

10. Guo, Z., Sun, H., Zhu, H.: Greedy construction of 2-approximation minimum Manhattan network. In: S. Hong, H. Nagamochi, T. Fukunaga (eds.) Proc. 19th Annu. Internat. Sympos. Algorithms Comput. (ISAAC'08), Lecture Notes Comput. Sci., vol. 5369, pp. 4-15. Springer, Berlin (2008). URL http://dx.doi.org/10.1007/ 978-3-540-92182-0_4

11. Kato, R., Imai, K., Asano, T.: An improved algorithm for the minimum Manhattan network problem. In: P. Bose, P. Morin (eds.) Proc. 13th Annu. Internat. Sympos. Algorithms Comput. (ISAAC'02), Lecture Notes Comput. Sci., vol. 2518, pp. 344-356. Springer, Berlin (2002). URL http://link.springer.de/link/service/series/ 0558/bibs/2518/25180344.htm

12. Lam, F., Alexandersson, M., Pachter, L.: Picking alignments from (Steiner) trees. J. Comput. Biol. 10, 509-520 (2003)

13. Lu, B., Ruan, L.: Polynomial time approximation scheme for the rectilinear Steiner arborescence problem. J. Comb. Optim. 4(3), 357-363 (2000)

14. Muñoz, X., Seibert, S., Unger, W.: The minimal Manhattan network problem in three dimensions. In: S. Das, R. Uehara (eds.) Proc. 3rd Internat. Workshop Algorithms Comput. (WALCOM'09), Lecture Notes Comput. Sci., vol. 5431, pp. 369-380. Springer, Berlin (2009). URL http://dx.doi.org/10.1007/978-3-642-00202-1_32

15. Nouioua, K.: Enveloppes de Pareto et réseaux de Manhattan: Caractérisations et algorithmes. Ph.D. thesis, Université de la Méditerranée (2005). URL http://www.lif-sud.univ-mrs.fr/ karim/download/THESE_NOUIOUA. pdf

16. Seibert, S., Unger, W.: A 1.5-approximation of the minimal Manhattan network problem. In: X. Deng, D. Du (eds.) Proc. 16th Intern. Symp. Algorithms Comput. (ISAAC'05), Lecture Notes Comput. Sci., vol. 3827, pp. 246-255. Springer, Berlin (2005). URL http://dx.doi.org/10.1007/11602613_26

17. Soto, J.A., Telha, C.: Jump number of two-directional orthogonal ray graphs. In: O. Günlük, G. Woeginger (eds.) Proc. 16th Internat. Conf. Integer Prog. Comb. Optimization (IPCO'11), Lecture Notes Comput. Sci., vol. 6655, pp. 389-403. Springer, Berlin (2011). URL http://dx.doi.org/10.1007/978-3-642-20807-2_31

18. Zelikovsky, A.: A series of approximation algorithms for the acyclic directed Steiner tree problem. Algorithmica 18(1), 99-110 (1997) 


\section{Appendix: Extension to Higher Dimensions}

We now describe the approximation algorithm for $d \mathrm{D}-\mathrm{MMN}$, for $d>3$, as a generalization of the 3D-MMN idea from Section 4.

Theorem 5. For any fixed dimension $d$ and for any $\varepsilon>0$, there exists an $O\left(n^{\varepsilon}\right)$-approximation algorithm for $d D-M M N$.

Large parts of the algorithm and the analysis are straightforward generalizations of the algorithm for 3D-MMN. The presentation of both follows closely the 3D case. However, Lemma 11, where the cost of the patching procedure is bounded, requires non-trivial additional insights.

As in the $3 \mathrm{D}$ case we decompose the overall problem into a constant number of instances of the directional subproblem. The directional subproblem consists in M-connecting any terminal pair $\left(t, t^{\prime}\right)$ such that $x^{i}(t) \leq x^{i}\left(t^{\prime}\right)$ for any $i \in\{1, \ldots, d\}$. Here, we use $x^{i}(p)$ to denote the $i$-th coordinate of point $p \in \mathbb{R}^{d}$. We can decompose the general problem into $2^{d-1}$ directional subproblems. Once again, we assume that the terminals are in general position.

\section{The $d \mathrm{D}$ Grid Algorithm}

We begin the description with a high-level summary. To solve the directional $d \mathrm{D}-\mathrm{MMN}$ problem we place a $d$ D-grid which partitions the instance into cuboids and slabs. Terminal pairs lying in different slabs are handled by M-connecting each terminal to the corner of its cuboid and then using the edges of grid. Terminal pairs from the same slab are M-connected by applying the algorithm recursively to all slabs. Each slab contains only a constant fraction of the terminals.

Step 1: Partitioning into cuboids and slabs. Consider the bounding cuboid $C$ for the set $T$ of terminals and choose a large constant $c=d^{1 / \varepsilon}$. For each dimension $i \in\{1, \ldots, d\}$ we choose $c+1$ separating planes determined by values $x_{1}^{i}<\cdots<x_{c+1}^{i}$. Planes $x_{1}^{i}$ and $x_{c+1}^{i}$ coincide with the boundary of $C$ in dimension $i$. The separating planes for dimension $i$ partition $C$ into $c$ slabs $C_{j}^{i}$, where $j \in\{1, \ldots, c\}$. Slab $C_{j}^{i}$ is the set of points $p \in C$ such that $x_{i}^{j} \leq x_{i}(p) \leq x_{i}^{j+1}$. We place the separating planes so that each slab contains at most $n / c$ terminals. Altogether we have $d(c+1)$ separating planes.

Let $j_{1}, \ldots, j_{d} \in\{1, \ldots, c\}$. The subcuboids $C_{j_{1}, \ldots, j_{d}}$ is the set of points $p \in C$ such that $x_{j_{i}}^{i} \leq$ $x^{i}(p) \leq x_{j_{i}+1}^{i}$ for each $i \in\{1, \ldots, d\}$.

Consider the $i^{\text {th }}$ dimension, $i \in\{1, \ldots, d\}$ and integers $j_{k} \in\{2, \ldots, c\}$ for each $k \in\{1, \ldots, d\}-$ $\{i\}$. Let $s$ be the axis-parallel line segment that contains all points $p \in C$ such that $x^{k}(p)=x_{j_{k}}$ for each $k \neq i$. We call $s$ a grid segment for dimension $i$. The grid $\mathcal{G}$ is the set of all grid segments and there are $d(c-1)^{d-1}$ grid segments in $\mathcal{G}$.

Given $j_{k} \in\{2, \ldots, c\}$ for each $k \in\{1, \ldots, d\}$ we call $\left(x_{j_{1}}^{1}, \ldots, x_{j_{d}}^{d}\right)$ a grid point of $\mathcal{G}$ and there are $(c-1)^{d}$ grid points in total.

Step 2: Add M-paths between different slabs. Consider two cuboids $C_{j_{1}, \ldots, j_{d}}$ and $C_{j_{1}^{\prime}, \ldots, j_{d}^{\prime}}$ with $j_{i}<j_{i}^{\prime}$ for each $i \in\{1, \ldots, d\}$. Any pair of terminals $t \in C_{j_{1}^{\prime}, \ldots, j_{d}^{\prime}}$ and $t^{\prime} \in C_{j_{1}^{\prime}, \ldots, j_{d}^{\prime}}$ can be M-connected using the segments of $\mathcal{G}$ as long as $t$ and $t^{\prime}$ are suitably connected to the corners (grid points) of their cuboids. We use patching (described below) to connect all terminal to the corners of their cuboid.

Patching: Call a cuboid $C_{j_{1}, \ldots, j_{d}}$ relevant if there is a cuboid $C_{j_{1}^{\prime}, \ldots, j_{d}^{\prime}}$ that contains at least one terminal and satisfies $j_{i}<j_{i}^{\prime}$ for each $i \in\{1, \ldots, d\}$. For each relevant cuboid $C_{j_{1}, \ldots, j_{d}}$, let $p_{j_{1}, \ldots, j_{d}}$ 
denote the grid point $\left(x_{j_{1}+1}^{i}, \ldots, x_{j_{d}+1}^{d}\right)$. Up-patching $C_{j_{1}, \ldots, j_{d}}$ means to M-connect every terminal in $t \in C_{j_{1}, \ldots, j_{d}}$ to $p_{j_{1}, \ldots, j_{d}}$. Up-patch $C_{j_{1}, \ldots, j_{d}}$ by solving the $d$ D-RSA problem with the terminals inside $C_{j_{1}, \ldots, j_{d}}$ as the terminals and $p_{j_{1}, \ldots, j_{d}}$ as the origin.

Down-patching is defined analogously; cuboid $C_{j_{1}, \ldots, j_{d}}$ is relevant if there is a non-empty cuboid $C_{j_{1}^{\prime}, \ldots, j_{d}^{\prime}}$ with $j_{i}^{\prime}<j_{i}, i=1, \ldots, d$ and using grid point $p_{j_{1}, \ldots, j_{d}}^{\prime}:=\left(x_{j_{1}+1}^{i}, \ldots, x_{j_{d}+1}^{d}\right)$ as origin instead of $p_{j_{1}, \ldots, j_{d}}$.

The output of this step is the union of grid $\mathcal{G}$ with a network that up-patches and down-patches all relevant cuboids. This produces M-paths between all terminal pairs in different slabs.

Step 3: Add M-paths within slabs. To also connect terminal pairs that lie in a common slab we apply the algorithm (Steps 1-3) recursively to each slab $C_{j}^{i}$ with $i \in\{1, \ldots, d\}$ and any $j \in\{1, \ldots, c\}$.

\section{Analysis}

We now show that the algorithm presented above yields a feasible solution to directional $d \mathrm{D}-\mathrm{MMN}$, with cost at most $O\left(n^{\varepsilon}\right)$ OPT, for any $\varepsilon>0$. Here, OPT denotes the cost of an optimum solution to the general $d \mathrm{D}-\mathrm{MMN}$ instance rather than the minimum cost $\mathrm{OPT}^{\prime}$ achievable for the directional subproblem. The reason is that the cost of the grid $\mathcal{G}$ is generally not related to $\mathrm{OPT}^{\prime}$ but to OPT. We finish the section by arguing that the running time of the algorithm is $n^{O(1 / \varepsilon)}$.

Lemma 9 (Feasibility). The $d D$ grid algorithm M-connects all relevant terminal pairs.

Proof. Let $\left(t, t^{\prime}\right)$ be a relevant terminal pair. First suppose that $t \in C_{j_{1}, \ldots, j_{d}}$ and $t^{\prime} \in C_{j_{1}^{\prime}, \ldots, j_{d}^{\prime}}$ where $j_{i}<j_{i}^{\prime}$ for all $i \in\{1, \ldots, d\}$. Hence, $C_{j_{1}, \ldots, j_{d}}$ and $C_{j_{1}^{\prime}, \ldots, j_{d}^{\prime}}$ are relevant for up-patching and downpatching, respectively. Consider the corners $p_{j_{1}, \ldots, j_{d}}$ of $C_{j_{1}, \ldots, j_{d}}$ and the corner $p_{j_{1}^{\prime}, \ldots, j_{d}^{\prime}}^{\prime}$ of $C_{j_{1}^{\prime}, \ldots, j_{d}^{\prime}}$. In the up-patching step of our algorithm we solve an RSA problem with terminals of $C_{j_{1}, \ldots, j_{d}}$ as the input points, and corner $p_{j_{1}, \ldots, j_{d}}$ as the origin. By definition, an RSA solution M-connects $t$ to $p_{j_{1}, \ldots, j_{d}}$. Similarly, down-patching M-connects $t^{\prime}$ to $p_{j_{1}^{\prime}, \ldots, j_{d}^{\prime}}^{\prime}$. It follows that $t$ and $t^{\prime}$ are connected, since $p_{j_{1}, \ldots, j_{d}}$ and $p_{j_{1}^{\prime}, \ldots, j_{d}^{\prime}}^{\prime}$ are M-connected via grid $\mathcal{G}$. Both terminals are even M-connected since additionally $t \leq p_{j_{1}, \ldots, j_{d}} \leq p_{j_{1}^{\prime}, \ldots, j_{d}^{\prime}}^{\prime} \leq t^{\prime}$, where $\leq$ denotes the domination relation between points.

Now suppose $t$ and $t^{\prime}$ lie in the same slab. As the algorithm is applied recursively to each slab there will be a recursion step where $t$ and $t^{\prime}$ will lie in cuboids in different slabs. Here, we need our assumption of general position. Applying the argument above to that particular recursive step completes the proof.

Approximation ratio. Let $r(n)$ denote the approximation ratio of our algorithm where $n$ is the number of terminals in $T$. The total cost of our solution consists of the cost for the grid $\mathcal{G}$, the cost of up-patching and down-patching all relevant cuboids, and the cost for the recursive treatment of the slabs in all $d$ dimensions. We analyze each of these costs separately.

The grid $\mathcal{G}$ consists of the $d(c-1)^{d-1}$ grid segments. The length of any grid segment $s$ is a lower bound on OPT. This holds because there are two terminals on the boundary of $C$ whose $L_{1}$-distance is at least the length of $s$. It follows that the cost of the grid is bounded by $d(c-1)^{d-1} \mathrm{OPT}$.

Let $r_{\text {patch }}(n)$ denote the cost of patching all relevant cuboids as is done in Step 2. Lemma 10 (given below) proves that $r_{\text {patch }}(n)=O\left(n^{\varepsilon}\right) \mathrm{OPT}$.

Now consider the recursive application of the algorithm to all slabs $C_{j}^{i}$, where $i \in\{1, \ldots, d\}$ and $j \in\{1, \ldots, c\}$. First recall that we placed the separating planes so that $\left|C_{j}^{i}\right| \leq n / c$ for any $i \in\{1, \ldots, d\}$ and any $j \in\{1, \ldots, c\}$. 
Consider dimension $i \in\{1, \ldots, d\}$. Let $\mathrm{OPT}_{j}^{i}$ be the optimum cost for M-connecting all (not only relevant) terminal pairs in slab $C_{j}^{i}$, where $j \in\{1, \ldots, c\}$. Slightly abusing notation, we write $N_{\text {opt }} \cap C_{j}^{i}$ for the set of line segments of $N_{\text {opt }}$ that are completely contained in the slab $C_{j}^{i}$. Observe that $N_{\text {opt }} \cap C_{j}^{i}$, forms a feasible solution for $C_{j}^{i}$. Thus $\mathrm{OPT}_{j}^{i} \leq\left\|N_{\mathrm{opt}} \cap C_{j}^{i}\right\|$. Each such $C_{j}^{i}$ contains at most $n / c$ terminals, and therefore the total cost of the solutions for the all slabs $C_{j}^{i}$ of dimension $i$ is at most

$$
\sum_{j=1}^{c} r\left(\frac{n}{c}\right) \mathrm{OPT}_{j}^{i} \leq r\left(\frac{n}{c}\right) \sum_{j=1}^{c}\left\|N_{\mathrm{opt}} \cap C_{j}^{i}\right\| \leq r\left(\frac{n}{c}\right) \mathrm{OPT} .
$$

Summing all costs, we obtain the following recursive equation for $r(n)$

$$
r(n) \mathrm{OPT} \leq d c^{d-1} \cdot \mathrm{OPT}+d \cdot r\left(\frac{n}{c}\right) \mathrm{OPT}+r_{\mathrm{patch}}(n) \mathrm{OPT} .
$$

Hence $r(n)=O\left(n^{\max \left\{\varepsilon, \log _{c} d\right\}}\right)$. Choosing $c \geq d^{1 / \varepsilon}$, as in Step 1, yields $O\left(n^{\varepsilon}\right)$ proving the approximation ratio claimed in Theorem 4 .

Lemma 10. The cost of patching all relevant cuboids, $r_{\text {patch }}(n)$, is $O\left(n^{\varepsilon}\right) \mathrm{OPT}$.

Proof. First consider up-patching. Lemma 11 (below) shows the existence of a near optimal network that up-patches all relevant cuboids. Lemma 12 shows that by reducing the patching problem to $d \mathrm{D}-\mathrm{RSA}$, we can find such a network of cost $O(\rho) \mathrm{OPT}$, where $\rho$ is the approximation factor of $d \mathrm{D}-\mathrm{RSA}$.

Analogously to the $3 \mathrm{D}$-case there is a approximation-preserving reduction from $d \mathrm{D}-\mathrm{RSA}$ to DST (see Section 2.3), which implies that $d \mathrm{D}$-RSA is approximable within a factor $O\left(n^{\varepsilon}\right)$ for any $\varepsilon>0$. Hence the same approximation factor can be achieved for $d \mathrm{D}$-RSA by choosing $\epsilon$ sufficiently small.

The lemma follows as the analysis holds analogously for down-patching.

Lemma 11. There exists an M-network of total cost at most $(c+1)^{d} \mathrm{OPT}$ that up-patches all relevant cuboids.

Proof. Let $I \subseteq\{1, \ldots, d\}$ be a set of dimensions. For every $i \in I$ we choose a separating plane $x_{j_{i}}^{i}$ where $j_{i} \in\{1, \ldots, c\}$. Let $J$ be the set of these separating planes and let $C(J)$ be the intersection of $C$ with all separating planes in $J$. We call $C(J)$ a $C$-face. There are most $(c+1)^{d}$ such $C$-faces. Project $N_{\text {opt }}$ onto each $C$-face. Let $N^{\prime}$ be the union of all these projections. Clearly, the cost of $N^{\prime}$ is at most $(c+1)^{d} \mathrm{OPT}$.

We claim that $N^{\prime}$ up-patches all relevant cuboids. To this end, let $\left(t, t^{\prime}\right)$ be a relevant terminal pair such that $t \in C_{j_{1}, \ldots, j_{d}}, t^{\prime} \in C_{j_{1}^{\prime}, \ldots, j_{d}^{\prime}}$ and $j_{i}<j_{i}^{\prime}$ for all $i \in\{1, \ldots, d\}$.

We claim that there is an M-path $\pi_{t}$ from $t$ to $p_{j_{1}, \ldots, j_{d}}$ in $N^{\prime}$. To see this, traverse the M-path $\pi_{t t^{\prime}}$ in $N_{\text {opt }}$ connecting $t$ and $t^{\prime}$, starting from $t$. We assume w.l.o.g. that the separating planes that bound cuboid $C_{j_{1}, \ldots, j_{d}}$ are entered by $\pi_{t t^{\prime}}$ in the order $\left(x_{j_{1}+1}^{1}, \ldots, x_{j_{d}+1}^{d}\right)$. The desired path $\pi_{t}$ starts at $t$ and follows $\pi$ until the separating plane $x_{j_{1}+1}^{1}$ is entered. From this point on we follow the projection $\pi_{t t^{\prime}}\left(x_{j_{1}+1}^{1}\right)$ of $\pi_{t t^{\prime}}$ onto $C$-face $C\left(x_{j_{1}+1}^{1}\right)$. If $\pi_{t t^{\prime}}\left(x_{j_{1}+1}^{1}\right)$ enters $x_{j_{2}+1}^{2}$ we follow the projection $\pi_{t t^{\prime}}\left(x_{j_{1}+1}^{1}, x_{j_{2}+1}^{2}\right)$ of $\pi_{t t^{\prime}}$ onto $\left.C\left(x_{j_{1}+1}^{1}, x_{j_{2}+1}^{2}\right)\right)$. We proceed in this fashion until we reach the $C$-face $C\left(x_{j_{1}+1}^{1}, \ldots, x_{j_{d}+1}^{d}\right)$, which is just the corner $p_{j_{1}, \ldots, j_{d}}$. Since $N^{\prime}$ contains the projection of $\pi_{t t^{\prime}}$ onto each $C$-face, the path $\pi_{t}$ described above is contained in $N^{\prime}$. This reasoning remains valid if the separating planes that bound $C_{j_{1}, \ldots, j_{d}}$ are entered in an arbitrary order, as we projected $N_{\mathrm{opt}}$ onto each $C$-face. 
Lemma 12. Given an efficient algorithm that approximates $d D-R S A$ within a factor of $\rho$, we can efficiently up-patch all relevant cuboids at cost at most $(2(c+1))^{d} \rho \mathrm{OPT}$.

Proof. In Lemma 11, we showed the existence of a network $N^{\prime}$ that up-patches all relevant cuboids at low cost. Now consider an arbitrary relevant cuboid $C_{j_{1}, \ldots, j_{d}}$. Clearly $N^{\prime} \cap C_{j_{1}, \ldots, j_{d}}$ up-patches $C_{j_{1}, \ldots, j_{d}}$. Hence $\mathrm{OPT}_{j_{1}, \ldots, j_{d}}^{\mathrm{up}} \leq\left\|N^{\prime} \cap C_{j_{1}, \ldots, j_{d}}\right\|$, where $\mathrm{OPT}_{j_{1}, \ldots, j_{d}}^{\mathrm{up}}$ denotes the cost of a minimum up-patching of $C_{j_{1}, \ldots, j_{d}}$. The problem of optimally up-patching $C_{j_{1}, \ldots, j_{d}}$ is just an instance $I_{j_{1}, \ldots, j_{d}}$ of $d \mathrm{D}-\mathrm{RSA}$, in which all terminals in $C_{j_{1}, \ldots, j_{d}}$ have to be connected by an M-path to $p_{j_{1}, \ldots, j_{d}}$. Applying the factor- $\rho$ approximation algorithm for $d \mathrm{D}$-RSA to each instance $I_{j_{1}, \ldots, j_{d}}$ with $C_{j_{1}, \ldots, j_{d}}$ relevant, we patch at total cost at most

$$
\rho \sum_{C_{j_{1}, \ldots, j_{d}} \text { relevant }} \mathrm{OPT}_{j_{1}, \ldots, j_{d}}^{\mathrm{up}} \leq \rho \sum_{C_{j_{1}, \ldots, j_{d}} \text { relevant }}\left\|N^{\prime} \cap C_{j_{1}, \ldots, j_{d}}\right\| \leq 2^{d} \rho\left\|N^{\prime}\right\| .
$$

The last inequality follows from the fact that each segment of $N^{\prime}$ occurs in at most $2^{d}$ cuboids. The lemma follows since $\left\|N^{\prime}\right\| \leq(c+1)^{d} \mathrm{OPT}$.

Running time. Let $T(n)$ denote the running time of the algorithm for $n$ terminals. The running time is dominated by patching and the recursive treatment of slabs. Using the DST algorithm of Charikar et al. 4], patching cuboid $C_{j}^{i}$ requires time $\left(n_{j}^{i}\right)^{O(1 / \varepsilon)}$, where $n_{j}^{i}$ is the number of terminals in $C_{j}^{i}$. As each cuboid is patched at most twice and there are $c^{d}$ cuboids, patching requires total time $O\left(c^{d}\right) n^{O(1 / \varepsilon)}=n^{O(1 / \varepsilon)}$. The algorithm is applied recursively to $d c$ slabs. This yields the recurrence $T(n)=d c T(n / c)+n^{O(1 / \varepsilon)}$, which leads to the claimed running time. 